\title{
Analysis of the Cellular Composition of the Arterial Intima with Modified En Face Techniques
}

\author{
Gunda Millonig, Harald Niederegger, and Georg Wick \\ Institute for Biomedical Aging Research (GM, GW), Austrian Academy of Sciences, Innsbruck, and Institute for \\ General and Experimental Pathology (GM, HN, GW), University of Innsbruck, Innsbruck, Austria
}

$T$ he innermost layers of blood vessels consist of a monolayer of endothelial cells sitting on a basement membrane with subendothelial connective tissue underneath. The lamina elastica interna forms the border of the muscular layer of the vessel wall, the media. The intima and its endothelial lining, respectively, are responsible for the inner smooth surface, the anticoagulant characteristics of the vessel wall, and the expression of adhesion molecules leading to infiltration of leukocytes modulated by the intimal extracellular matrix and is therefore involved in initiation of immunological-inflammatory reactions. Atherosclerosis is one possible consequence of such an inflammatory reaction of the arterial wall, showing typical features of inflammation in early stages induced by cellular components (Ross, 1993; WaltnerRomen et al, 1998; Wick et al, 1997; Xu et al, 1990) and cytokines (Tellides et al, 2000). In this respect, it is of great interest to study the exact morphology and composition of the intimal architecture. Although it is not difficult to stain the cells of interest with immunohistochemical methods on conventional frozen or paraffin-embedded sections, these 4- $\mu \mathrm{m}$-thick sections can only be regarded as snapshots, representing the local situation within these $4 \mu \mathrm{m}$. In-depth information about cell distribution over the whole vessel requires the production of serial sections, which is a time-consuming and expensive process and also renders result interpretation difficult, because cells or cell processes are sliced and appear in several sections, tempting the observer to count them more than once. Another disadvantage of conventional evaluation techniques is the difficulty in orienting the position of the cells toward each other.

To overcome these handicaps, the intima can be prepared as a thin membrane specimen (Häutchen preparation or, as we call it, the "intimal sheet"). This technique has been used since the 1960s, but only in

Received December 22, 2000.

This study was supported by the Austrian Science Fund, Project No. P-12213/MED (to GW), the Hans und Blanca Moser-Stiftung (to GM) and a "Förderungs-stipendium" of the University of Innsbruck (to GM). Address reprint requests to: Dr. Georg Wick, Institute for Biomedical Aging Research, Austrian Academy of Sciences, Rennweg 10, 6020 Innsbruck, Austria.E-mail:Georg.Wick@uibk.ac.at rare occasions. We now propose different methods for the preparation of arterial intimal sheets in human and mouse arteries, which bring excellent results after indirect immunofluorescence staining and examination in the laser confocal scanning microscope. This method improves the possibility of observing cells in their natural habitat without destroying them by sectioning. Intimal sheets permit investigation of differences in the intimal composition between atherosclerosis-prone (aortic arch and bifurcational areas, where turbulent predominates over laminar flow patterns) and relatively atherosclerosis-resistant regions (straight parts of the artery subjected to laminar flow conditions). To guarantee that the separation took place along the lamina elastica interna, we performed controls by cutting frozen sections of the intimal sheets. Intact endothelium was proven by staining for von Willebrand Factor.

For this short methodological communication, we chose to compare mouse (Fig. 1) and human intima (Fig. 2) for the presence of major histocompatibility complex (MHC) class $\mathrm{II}^{+}$cells. For these experiments, we used arterial specimens from C57BL/6 mice and humans. C57BL/6 mice were obtained from Charles River (Sulzfeld, Germany), housed in groups of 5 to 12, and fed a standard diet. At the age of 6 to 10 weeks, they were killed under pentobarbital anesthesia by the removal of heart and aorta.

Aortae were carefully removed from the aortic arch to the bifurcation into the two iliac arteries, opened longitudinally, and rinsed in phosphate buffered saline (PBS, pH 7.2). All procedures took place under an stereomicroscope.

Human arterial samples were taken from the carotid arteries, aortae, and iliac arteries of individuals whose deaths were due to suicide, homicide, or accident (obtained from the Department of Forensic Medicine, University of Innsbruck Medical School, Innsbruck, Austria), or who were candidates for organ explantation (obtained from the Department of Transplant Surgery, University of Innsbruck Medical School) and who did not suffer from any clinical manifestation of cardiovascular disease.

In humans, the intima was stable enough to be prepared before staining procedures started. We modified a protocol known from dermatological research for the preparation of epidermal sheets, in 


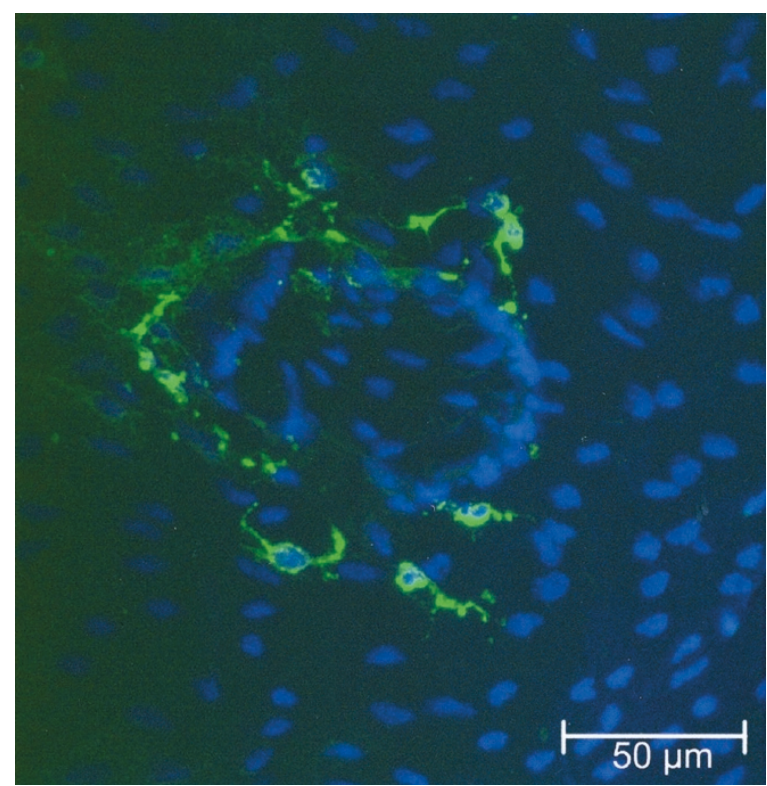

Figure 1.

Mouse intimal sheet stained in indirect immunofluorescence for major histocompatibility complex (MHC) class $\mathrm{II}^{+}$cells (green). En face staining allows for assessment of the cellular distribution in the observed area as shown in this example where MHC class $\mathrm{II}^{+}$cells accumulate around the ostium of the mesenteric artery. Nuclei are counterstained in blue 4',6diamidino-2-phenylindole (4') (DAPI).

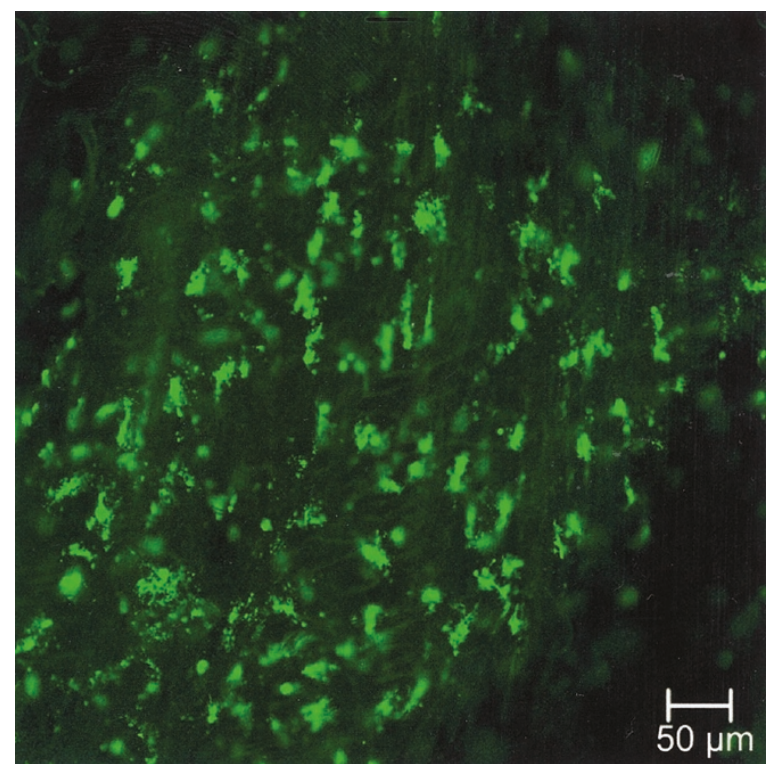

Figure 2.

Human aortic intimal sheet stained in indirect immunofluorescence for MHC class $\mathrm{II}^{+}$cells. The specimen was incubated overnight with the primary antibody followed by extensive washing. The secondary antibody was FITCconjugated, and $\mathrm{MHC}$ class $\mathrm{II}^{+}$cells are stained in green.

which the epidermis is separated from the dermis (Juhlin and Shelley, 1977). The longitudinally opened aortae were dissected into small pieces of approximately $5 \times 5 \mathrm{~mm}$ with small, sharp scissors followed by incubation in $0.5 \mathrm{M} \mathrm{NH}_{4} \mathrm{SCN}$ (Merck, Darmstadt, Germany) at $37^{\circ} \mathrm{C}$ for 60 minutes. The arterial pieces were numbered, and the numbers were documented in

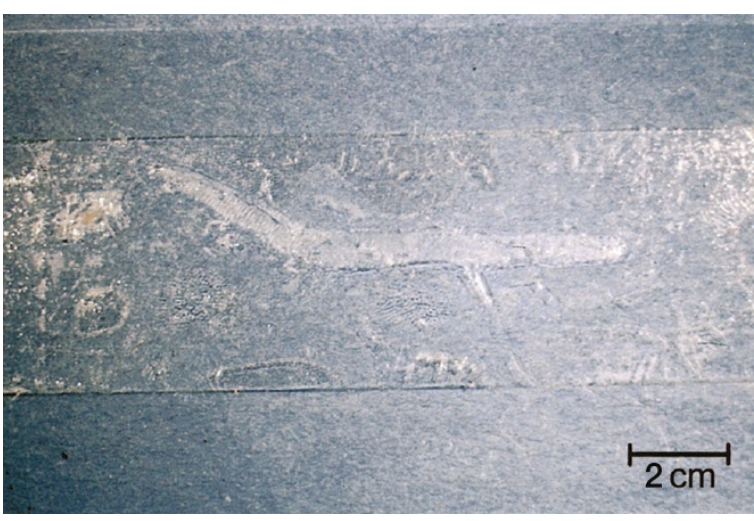

Figure 3.

Macroscopic view of a mouse aortic intimal sheet attached to a glass slide with double-sided tape.

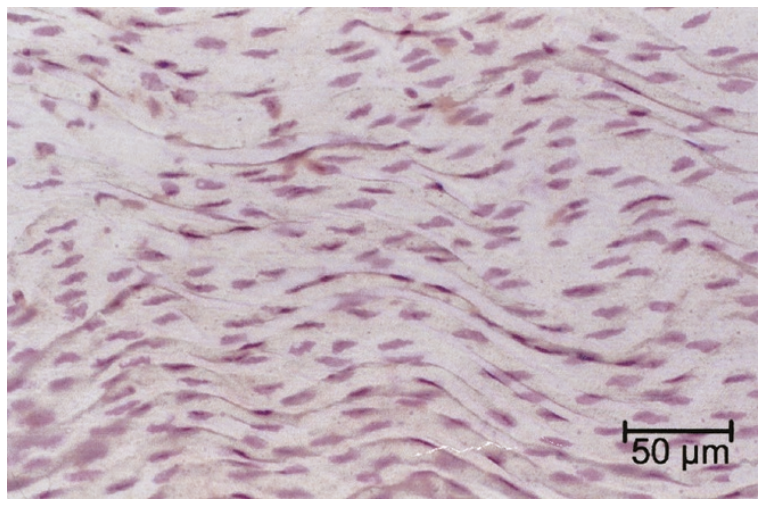

Figure 4.

The same mouse aortic intimal sheet as in Figure 3 stained with hematoxylin Nuclei of endothelial cells are stained and show a regular streamlined pattern representing a continuous endothelial layer unaffected by the preparation procedure.

a sketch of the arterial bifurcation to indicate whether a sheet was prepared from an atherosclerosis-prone area or not. After washing in PBS at room temperature, the arterial piece was placed on a Petri dish, endothelial side up, and the intima was gently lifted from the rest of the vessel wall with delicate forceps. Best results were obtained by picking the intima at one edge and slowly pulling it from the rest of the vessel wall, grasping the intima as closely as possible to the point where the intima was lifted off. After rinsing in PBS for 30 minutes, the sheets were ready for staining. Sheets were acetonefixed for 10 minutes at room temperature and then rinsed in PBS for 30 to 60 minutes. Incubation in the optimally diluted primary antibody followed overnight at $4^{\circ} \mathrm{C}$ (human: mouse anti-HLA-DR/DP/DQ [DAKO, Glostrup, Denmark]; mouse: hamster anti-mouse MHC II, clone N22 [a gift from N. Romani, Department of Dermatology, University of Innsbruck Medical School]). For all experiments, negative controls were carried out, either by omitting the first antibody or by using an irrelevant isotype-matched antibody. Washing was done in $\mathrm{PBS} / 1 \%$ bovine serum albumin (BSA; Sigma, Munich, Germany) for 4 hours at room temperature on a multiaxle rotator, and the sheet was incubated in diluted fluorochrome-labeled secondary antibody for the next 4 
hours at room temperature (human: rabbit anti-mouseFITC, [DAKO]; mouse: goat anti-hamster-ALEXA568 [Molecular Probes, Eugene, Oregon]). Finally, the sheets were washed in PBS/BSA $1 \%$ on the rotator at room temperature overnight and mounted the following morning. Incubation in $\mathrm{NH}_{4} \mathrm{SCN}$, acetone fixation, and incubation with antibodies were performed in micro test tubes (Eppendorf-Netherler-Hinz, Hamburg, Germany), whereas the washing took place in $50 \mathrm{ml}$ tubes (Falcon; Becton Dickinson Labware, Franklin Lakes, New Jersey) to guarantee excess washing fluid.

Mouse aortae are so tiny that it was not possible to divide the intima from the rest of the vessel wall, as described above. Therefore, the staining procedure was done beforehand and the whole aorta was stained as described for the human aortae. Mouse aortae were removed and placed on a Petri dish with a drop of physiological saline. To obtain a straight longitudinal cut to open the vessel, glass Pasteur pipettes were pulled over a Bunsen burner (thin enough to be introduced into the mouse aorta and ending with a round tip to preserve the intact endothelium). The pipette was introduced into the aorta from the proximal part and served to straighten and fix the vessel, allowing a cut to be made along the vessel wall with microscissors. The longitudinally opened aorta underwent the staining procedure. After the last rinsing overnight, aortae were counterstained for 10 minutes with DAPI (4', 6-diamidino-2-phenylindole, Molecular Probes) (1:2000) and washed in PBS for another 10 minutes. Thereafter, they were immersed in pure alcohol for dehydration for 20 minutes and immediately pressed, endothelial side down, onto clear double-sided Scotch tape (3M, Cergy-Pontoise, France) on a glass slide. The tissue was allowed to dry for 10 to 15 minutes and was then rehydrated for 10 minutes with PBS containing $10 \%$ (v/v) glycerol. The media and adventitia were peeled away in a continuous motion using fine forceps, leaving the intima from the whole aorta in one piece on the tape (Figs. 3 and 4). Mounting in antifading mounting medium (Mowiol; Calbiochem-Novabiochem, Bad Soden, Germany) completed the procedure. All specimens were examined with a laser scanning confocal fluorescence microscope (Zeiss, Jena, Germany) using magnifications ranging between 100-fold and 630-fold.

\section{Acknowledgements}

We thank Dr. Hermann Dietrich for help with the animal experiments, Dr. Boris W. Hochleitner and Dr. Walter Rabl for the human arterial specimens, and Dr. Nikolaus Romani for the antibody-supernatant.

\section{References}

Juhlin L and Shelley WB (1977). New staining techniques for the Langerhans cell. Acta Derm Venereol 57:289-296.

Ross $R$ (1993). The pathogenesis of atherosclerosis: A perspective for the 1990s. Nature 362:801-809.

Tellides G, Tereb DA, Kirkiles-Smith NC, Kim RW, Wilson JH, Schechner JS, Lorber MI, and Pober JS (2000). Interferongamma elicits arteriosclerosis in the absence of leukocytes. Nature 403:207-211.

Waltner-Romen M, Falkensammer G, Rabl W, and Wick G (1998). A previously unrecognized site of local accumulation of mononuclear cells. The vascular-associated lymphoid tissue. J Histochem Cytochem 46:1347-1350.

Wick G, Romen M, Amberger A, Metzler B, Mayr M, Falkensammer G, and Xu Q (1997). Atherosclerosis, autoimmunity, and vascular-associated lymphoid tissue. FASEB J 11:11991207.

Xu QB, Oberhuber G, Gruschwitz M, and Wick G (1990). Immunology of atherosclerosis: Cellular composition and major histocompatibility complex class II antigen expression in aortic intima, fatty streaks, and atherosclerotic plaques in young and aged human specimens. Clin Immunol Immunopathol 56:344-359. 